\title{
MPI communication benchmarking on Intel Xeon dual quad-core processor cluster
}

\begin{abstract}
This paper reports the measurements of MPI communication benchmarking on Khaldun cluster which ran on Linux-based IBM Blade HS21 Servers with Intel Xeon dual quad-core processor and Gigabit Ethernet interconnect. The measurements were done by using SKaMPI and IMB benchmark programs. Significantly, these were the first results produced by using SKaMPI and IMB to analyze the performance of Open MPI implementation on Khaldun cluster. The comparison and analysis of the results of point to point and collective communication from these two benchmark programs were then provided. It showed that different MPI benchmark programs rendered different results since they used different measurement techniques. The results were then compared to the experiment's results that were done on cluster with Opteron dual quad-core processor and Gigabit Ethernet interconnect. The analysis indicated that the architecture of machines used also affected the results.
\end{abstract}

Keyword: MPI benchmarks; Parallel computer; IBM Blade HS21 server; Multi-core; SKaMPI; IMB 